\title{
Status of the RHIC Spin Program
}

\author{
L.C. Bland \\ Brookhaven National Laboratory, Upton, NY USA
}

\begin{abstract}
The Relativistic Heavy Ion Collider (RHIC) at Brookhaven National Laboratory has been developing the capability of accelerating, storing and colliding high-energy polarized proton beams over the past several years. During this development phase, important first measurements of cross sections and spin asymmetries for neutral pions produced in polarized proton collisions at $\sqrt{s}=200$ $\mathrm{GeV}$ have been completed by STAR and PHENIX, the two large collider experiments at RHIC. This contribution reports on progress of the RHIC spin program and provides an outlook for the future.
\end{abstract}

\section{INTRODUCTION}

To date, our knowledge of how the spin of the proton is distributed amongst its quark and gluon constituents comes from studies of deep-inelastic scattering (DIS) of polarized electrons and muons from polarized proton, deuteron and ${ }^{3} \mathrm{He}$ targets. Global analyses of this data (e.g., Ref. [1] and references to the data therein) have provided a map of the Bjorken- $x$ dependence of the quark (and anti-quark) polarization. These studies have left us with many puzzles, the most significant of which is how the proton gets its spin from its constituents. The spin physics program at the Relativistic Heavy Ion Collider (RHIC) at Brookhaven National Laboratory [2] is an ambitious effort to address the puzzles by measuring spin asymmetries of particles produced in polarized proton collisions at center of mass energy in the range $200 \leq \sqrt{s} \leq 500 \mathrm{GeV}$. The object is to utilize polarized quarks as a probe of the polarization of the constituents of the proton in scattering processes that are described by perturbative QCD. To achieve this objective, it is necessary to establish that pQCD quantitatively describes experimental cross sections and particle correlations at these collision energies.

One of the long term objectives of the RHIC spin program is to determine the Bjorken $x$ dependence of the helicity asymmetry distribution of gluons within a longitudinally polarized proton, $\Delta G(x)$. Completion of double helicity asymmetry $\left(A_{L L}\right)$ measurements for particles produced in different pseudorapidity $(\eta)$ ranges and as a function of transverse momentum $\left(p_{T}\right)$ can result in a detailed map of gluon polarization over a broad range of Bjorken $x$. Inclusive meson and jet production probes $\Delta G(x)$ through multiple partonic subprocesses with contributions from a broad range of $x_{\text {gluon }}$ as specified by the standard convolution integrals for hard scattering processes. Di-hadron and di-jet production can provide additional constraints on the kinematics, thereby changing the admixture of partonic subprocesses and providing tighter constraints on the Bjorken $x$ values of the partons that participate in the hard scattering. Prompt photon production is expected to be dominated by the QCD Compton process $(q g \rightarrow q \gamma)$, thereby providing a more selective probe of $\Delta G(x)$. Further information can be provided by the measure- 
ment of $A_{L L}$ for particle correlations, such as $\gamma+$ jet coincidences [3]. At $\sqrt{s}=500 \mathrm{GeV}$, measurements of the parity violating single spin asymmetry $A_{L}$ for $W^{ \pm}$production will result in the flavor separation of quark polarization at large Bjorken $x$ and anti-quark polarization at moderately small $x$, particularly when the daughter leptons from $W^{ \pm}$ decay are detected at large rapidity. The unknown transversity structure function can be probed via measurement of the transverse spin correlation coefficient $\left(A_{T T}\right)$ for inclusive jet production.

\section{HOW RHIC SPIN WORKS}

High-energy electrons develop transverse polarization in a storage ring because of a small spin flip probability in the sychrotron radiation they emit. Muons are produced through the decays of pion beams, and so are naturally polarized because the weak interaction violates parity. To provide significant polarization for intense proton beams at high energy, it is necessary to use atomic methods at an ion source and then preserve the polarization through the acceleration sequence. The development of Siberian snakes [4] has enabled the preservation of polarization while accelerating protons to high energies through numerous depolarizing resonances in synchrotrons. To date, the highest energy polarized proton beam produced in a synchrotron is at RHIC and is $100 \mathrm{GeV}$. Previously, the highest energy polarized proton collisions were studied using $200 \mathrm{GeV}$ proton beams in fixed target experiments. The beams were produced by the parity-violating decays of hyperons [5] and the total center-of-mass energy for those experiments was $\sqrt{s}=20$ $\mathrm{GeV}$. At RHIC, the polarized proton beams collide, enabling spin physics experiments at collision energies $200 \leq \sqrt{s} \leq 500 \mathrm{GeV}$. There are plans to accelerate polarized proton beams with energies greater than $200 \mathrm{GeV}$ in the upcoming RHIC run as part of the development of the capability for studying spin asymmetries for $W^{ \pm}$production. Exquisite care must be taken throughout the entire acceleration chain to ensure that the polarization is preserved. Significant progress has been made in the development of these capabilities in the first years of RHIC operations.

Also differing from polarized DIS experiments is the need to develop methods to measure the polarization of high energy polarized protons. Precision measurements of spin observables requires accurate determination of the beam polarization. For polarized lepton beams used in DIS, quantum electrodynamics can be used to accurately predict cross sections and spin observables for scattering processes. Spin dependent scattering processes involving polarized protons must be calibrated by experiment since robust theoretical calculations of spin observables at small momentum transfer, where interaction rates are large, cannot be reliably made from first principles.

The RHIC polarimeters are based on measuring proton elastic scattering from carbon at very small four momentum transfer, in the range $0.006 \leq-t \leq 0.03(\mathrm{GeV} / \mathrm{c})^{2}$ [6]. An analyzing power $\left(A_{N}\right)$ is expected because of the interference of the Coulomb spin-flip amplitude and the spin-indepedent strong interaction amplitude; hence, the polarimetry process is referred to as Coulomb-Nuclear Interference (CNI). The observed $-t$ dependence of the measured spin-dependent asymmetry, $P_{\text {beam }} \times A_{N}$, suggests substantial contributions from the hadronic spin flip amplitude, meaning that independent 
measurements are necessary to determine the beam polarization $\left(P_{\text {beam }}\right)$. Precision measurements were made for the first time at $100 \mathrm{GeV}$ in RHIC run 4 (April through May, 2004) by exploiting identical particle symmetries in polarized proton elastic scattering from a polarized gas jet target $[7,8]$. The contribution of the spin dependendent strong interaction amplitude to small $|t|$ elastic scattering is of interest in its own right, and can be extracted from spin observables measured at RHIC in either fixed target experiments with the polarized gas jet target or with carbon ribbon targets, or by studying elastic scattering of the polarized colliding beams [9], which is the object of the pp2pp experiment at RHIC.

The first polarized proton collisions at $\sqrt{s}=200 \mathrm{GeV}$ were achieved during RHIC run 2 (January through May, 2002). Typical collision luminosities were $\sim 0.5 \times 10^{30}$ $\mathrm{cm}^{-2} \mathrm{~s}^{-1}$ and the average polarization was $\sim 16 \%$, inferred from assumptions about the beam energy dependence of the CNI analyzing power [10]. Significant polarization loss in the RHIC injector resulted because the rate of acceleration in the AGS was two times smaller than expected because of equipment failure. An integrated luminosity of 0.3 $\mathrm{pb}^{-1}$ was observed at the STAR interaction point. Only vertical beam polarization was available.

Polarized proton collisions at $\sqrt{s}=200 \mathrm{GeV}$ were also achieved during RHIC run 3 (March through May, 2003). Typical collision luminosities observed with all subsystems operational at STAR were $3 \times 10^{30} \mathrm{~cm}^{-2} \mathrm{~s}^{-1}$ and the beam polarization averaged $\sim 25 \%$, based again on assumptions about the beam energy dependence of the effective analyzing power of the polarimeter. In this run, helical dipole magnets located either side of PHENIX and STAR were used to produce longitudinal polarization at the interaction points. Local polarimeters were developed by both experiments to establish the absence of transverse polarization components for the two beams at the interaction point concurrent with measurement of spin-dependent asymmetries by the CNI polarimeters that are located in parts of the ring where the beam polarization is vertical. The combined results from the local polarimeters and the CNI polarimeters established that the colliding beams at STAR and PHENIX had longitudinal polarization. Integrated luminosities of $0.5 \mathrm{pb}^{-1}$ with vertical polarization and $0.4 \mathrm{pb}^{-1}$ with longitudinal polarization were observed at the STAR interaction point.

Time was devoted during run 4 to accelerator development interleaved with the polarization calibration experiment. A new betatron tune for RHIC was developed to reduce beam loss induced by the influence of one beam on the other at the interaction points. With unpolarized beam, collision luminosities in excess of $10^{31} \mathrm{~cm}^{-2} \mathrm{~s}^{-1}$ were achieved during tests. It was also established that beam polarization could be preserved with the new tune of the accelerator. Commissioning of a new warm-bore partial helical snake in the AGS was successful. At the end of the run there was a successful demonstration of repeated stores in RHIC with an average collision luminosity of $4 \times 10^{30} \mathrm{~cm}^{-2} \mathrm{~s}^{-1}$ with average polarization in both beams of $\sim 45 \%$.

For the immediate future, a long polarized proton run is planned for the RHIC run starting in November, 2004. Performance comparable to that demonstrated during run 4 is expected to result in proton collisions with an average polarization of $45 \%$ and an average integrated luminosity of $1 \mathrm{pb}^{-1}$ per week at a collision energy of $\sqrt{s}=200 \mathrm{GeV}$. As discussed below, this should result in good measurements of $A_{L L}$ for inclusive $\pi^{0}$ and jet production, thereby providing sensitivity to possible gluon 
polarization. A new superconducting partial helical snake magnet for the AGS is planned to be commissioned during this run. It is anticipated that this device will ultimately permit full transmission of the polarization from the ion source into RHIC at the design intensity of $2 \times 10^{11}$ protons per bunch. Plans have been formulated to resolve the vacuum breakdown issue within RHIC which presently limits luminosity. When these developments are complete, it is expected that design luminosity and polarization for $\vec{p}+\vec{p}$ collisions will be realized.

\section{WHAT HAS BEEN LEARNED TO DATE?}

The first polarized proton collisions at RHIC at $\sqrt{s}=200 \mathrm{GeV}$ resulted in measurements of the cross section for neutral pion production at both PHENIX and STAR (Fig. 1). The results were for quite different $\pi^{0}$ rapidities, with PHENIX reporting the $p_{T}$ dependence of the cross section near $\eta \approx 0$ and STAR reporting the Feynman $x$ dependence of the cross section at $\langle\eta\rangle=3.3$ and $\langle\eta\rangle=3.8$. These data have been compared with state of the art perturbative QCD calculations [11] computed at next-to-leading order. The calculations use the CTEQ6M [12] parton distribution functions and the KKP [13] and Kretzer [14] fragmentation functions.

The theoretical calculations are found to quantitatively represent the measured cross sections at $\sqrt{s}=200 \mathrm{GeV}$ over a broad range of transverse momentum that extends down to $p_{T} \approx 1 \mathrm{GeV} / \mathrm{c}$. Agreement between calculation and data for transverse momenta between $1 \leq p_{T} \leq 10 \mathrm{GeV} / \mathrm{c}$ had also been observed for inclusive particle production at the Tevatron at much higher $\sqrt{s}$ [13]. The results from RHIC form part of a picture of the pseudorapidity and collision energy dependence of $\pi^{0}$ production. Comparison of NLO pQCD calculations to data obtained at lower $\sqrt{s}$ [17] shows a systematic tendency for more quantitative agreement as $\sqrt{s}$ increases. It is also the case that the agreement extends over an increasingly broader range of $\eta$ as $\sqrt{s}$ increases. As shown in Fig. 1, at RHIC collision energies, agreement between first principles calculations and measured cross sections extends over a broad rapidity interval. This agreement suggests that rapidity dependence of particle production can be exploited to significantly alter the admixture of $g g, q g$ and $q q^{\prime}$ subprocess contributions and to emphasize partonic contributions from different regions of Bjorken $x$.

Understanding $\pi^{0}$ production with the electromagnetic calorimeters in PHENIX and from STAR is also an important prerequisite for using these devices for prompt photon detection. We can anticipate results for cross sections [18] and spin observables for prompt photons in the near future.

In addition to inclusive cross sections, the first RHIC runs have produced results for particle correlations. At mid-rapidity, measurements of azimuthal correlations of pairs of charged hadrons produced at midrapidity have been reported for $p+p, d+A u$ and $\mathrm{Au}+\mathrm{Au}$ collisions at $\sqrt{s_{N N}}=200 \mathrm{GeV}$ [19]. For $\mathrm{p}+\mathrm{p}$ collisions the correlations have the pattern expected for a partonic scattering origin to high- $p_{T}$ particle production; namely, a relative narrow peak when the hadrons have a small azimuthal angle difference ('nearside') $(\Delta \phi)$ and a broader, but well developed, peak when they are separated by $\Delta \phi=$ $180^{\circ}$ ('away-side'). These studies have been extended to hadron pairs separated by large 


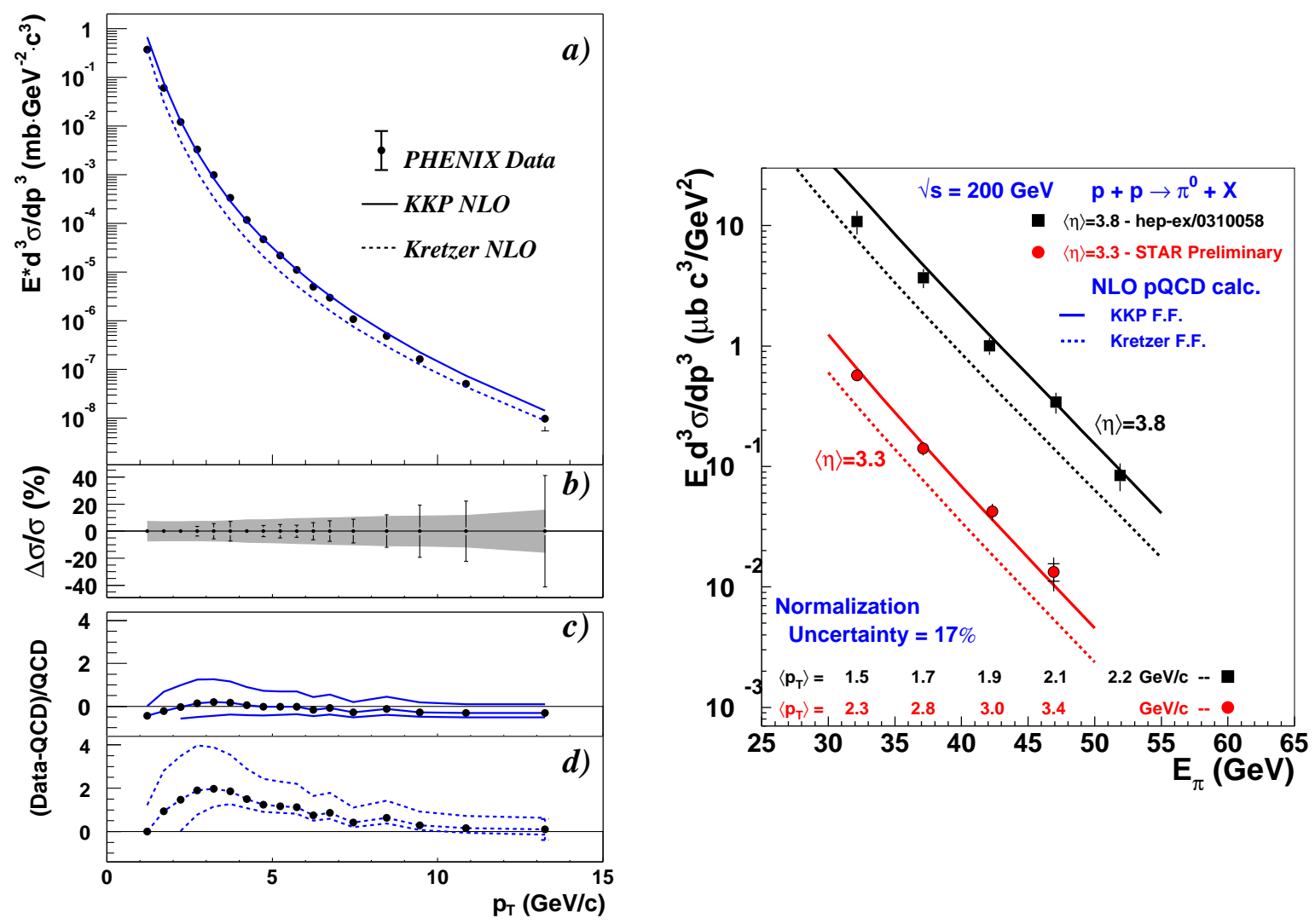

FIGURE 1. Results for (left) the invariant cross section for neutral pions produced at midrapidity by the PHENIX collaboration [15] and (right) the invariant cross section for neutral pions produced at large rapidity by the STAR collaboration [16] in $\mathrm{p}+\mathrm{p}$ collisions at $\sqrt{s}=200 \mathrm{GeV}$ in comparison with next-toleading order perturbative QCD calculations.

rapidity intervals [20]. Strong azimuthal correlations are observed for $\pi^{0}-h^{ \pm}$hadron pairs separated by four units of rapidity. To date, comparisons of azimuthal correlations with theoretical predictions have been limited. One analysis [21] has established the need for multiple-soft gluon emissions to understand the width and shape of the away-side dihadron $\Delta \phi$ distribution. It is fully anticipated that di-hadron correlations will provide a wealth of information when confronted against NLO pQCD calculations. Furthermore, future measurements of transverse and longitudinal spin observables for di-hadron, di-jet and $\gamma+$ jet events will lead to many interesting results.

The first measurements of spin observables in $\pi^{0}$ production have been made as RHIC develops the polarization and intensity of the colliding proton beams. For $\pi^{0}$ produced at large rapidity, a large analyzing power has been observed in transversely polarized proton collisions at $\sqrt{s}=200 \mathrm{GeV}$ [16] (left side of Fig. 2). The pQCD picture that describes the unpolarized cross section in these kinematics identifies the dominant partonic process as large Bjorken- $x$ quarks (from the polarized proton) interacting with soft gluons from the other proton. At large $x$, global analyses have established the quarks are highly polarized within a longitudinally polarized proton. If transversity behaves in a similar fashion, then the large $A_{N}$ could result from highly polarized quarks that undergo 

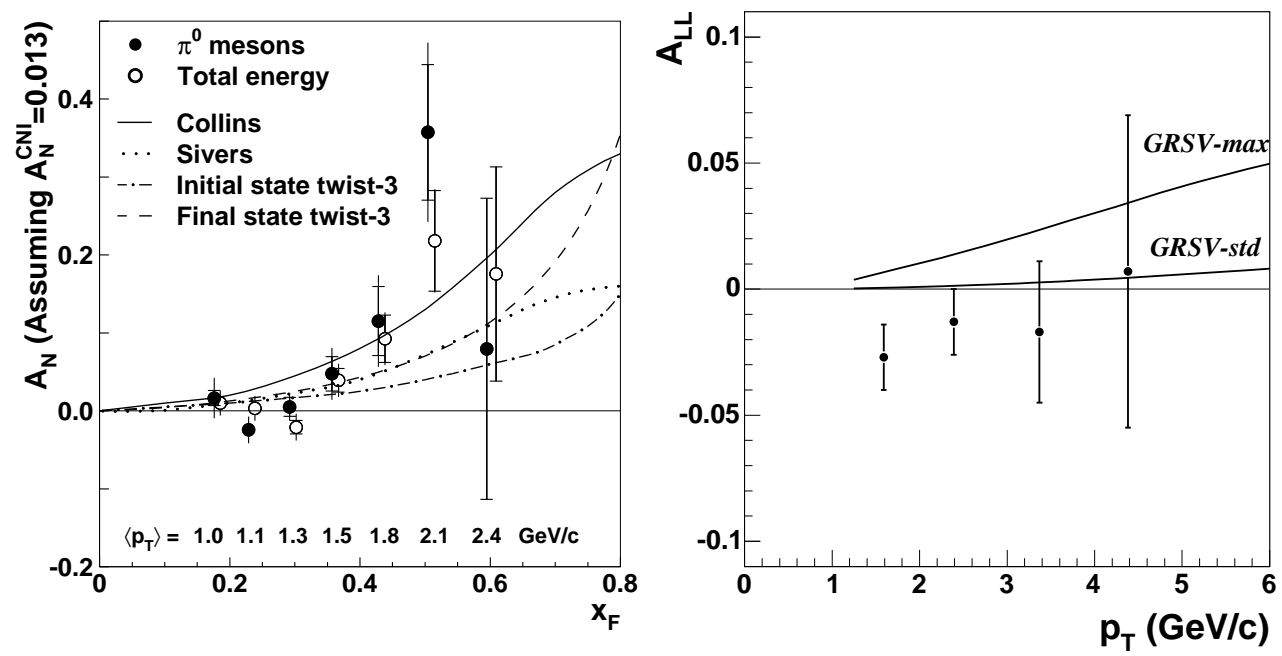

FIGURE 2. The first spin asymmetries from RHIC from $\vec{p}+\vec{p}$ collisions at $\sqrt{s}=200 \mathrm{GeV}$. (Left) The Feynman $x$ dependence of the analyzing power for large rapidity $\pi^{0}$ production in comparison to QCD model expectations. (Right) The $p_{T}$ dependence of the longitudinal spin asymmetry, $A_{L L}$ for $\pi^{0}$ production at midrapidity.

a spin- and transverse-momentum $\left(k_{T}\right)$ dependent fragmentation into $\pi^{0}$ (Collins effect) $[22,23]$. It could also be that the quarks involved in the particle production are from a spin- and $k_{T}$-dependent distribution function associated with the polarized proton (Sivers effect) $[24,25]$. There are also twist- 3 mechanisms that can give rise to the observed $A_{N}$ [26]. Further experiments are required to disentangle these mechanisms. Possibilities include the measurement of the spin dependence of azimuthally correlated hadron pairs $\left(\right.$ e.g.,$\left.\pi^{0}-h^{ \pm}\right)$. Extension of these measurements to negative $x_{F}$ could isolate contributions from the Sivers function associated with gluons, since gluons cannot contribute to transversity within the proton, thereby eliminating the Collins effect. It would also be useful to separately study the $x_{F}$ and $p_{T}$ dependence of $A_{N}$. We can also expect results from the BRAHMS experiment for the $x_{F}$ dependence of $A_{N}$ for charged pions [27].

The $A_{N}$ results for $\vec{p}+\vec{p}$ collisions at $\sqrt{s}=200 \mathrm{GeV}$ have an $x_{F}$ dependence quite similar to that observed at $\sqrt{s}=20 \mathrm{GeV}$ [28] and at lower energies [29]. One of the challenges is to obtain a robust theoretical understanding of the dynamics responsible for this phenomena at energies where NLO pQCD provides a good framework for understanding the cross section and at lower energies where the theory fails to explain data [17].

The right side of Fig. 2 shows the first measurment of $A_{L L}$ for $\pi^{0}$ production at RHIC, recently reported by the PHENIX collaboration [30]. Data from midrapidity $\pi^{0}$ and jet production promise to provide insight into gluon polarization. First measurements of $A_{L L}$ for midrapidity $\pi^{0}$ production in $\vec{p}+\vec{p}$ interactions at $\sqrt{s}=20 \mathrm{GeV}$ were made by the 
E704 collaboration [31]. The first $A_{L L}$ measurement at RHIC at $\sqrt{s}=200 \mathrm{GeV}$ is based on an integrated luminosity of only $0.22 \mathrm{pb}^{-1}$ with average beam polarization of $27 \%$. Even with these limited statistics the $\pi^{0} A_{L L}$ measurement has accuracy comparable to the uncertainty on gluon polarization deduced from inclusive DIS measurements, as reflected by the two curves in the figure. The GRSV-max curve is computed [32] assuming $100 \%$ gluon polarization at the input scale, $Q_{0}^{2}=0.6 \mathrm{GeV}^{2}$. The GRSV-std curve represents the best global fit [33] to the inclusive polarized DIS data. Given the improved performance of polarized proton operations at RHIC, there is promise for at least an order of magnitude improvement in the statistical accuracy of $A_{L L}$ in the upcoming RHIC run.

What does this mean for gluon polarization? Inclusive observables are computed as a convolution of distribution functions, hard scattering cross section and fragmentation functions. Consequently, $\Delta G(x)$ must be extracted from a global theoretical analysis, in a manner similar to that used for unpolarized parton distribution functions. The midrapidity data probes Bjorken $x$ values that are on average equal to $x_{T}=2 p_{T} / \sqrt{s}$. Tests of the resulting $\Delta G(x)$ can be made by measurements of the rapidity dependence of $A_{L L}$ for $\pi^{0}$ production; midrapidity inclusive jet production, that would eliminate the fragmentation function from the convolution integral; and di-hadron correlations, that can narrow the range of contributions from the distribution functions depending on the kinematics of the hadron pair. Measurements of $A_{L L}$ for prompt photon production are expected in the future, when the luminosity and polarization of the RHIC beams are further developed.

\section{SUMMARY AND OUTLOOK}

RHIC is the world's first polarized proton collider. The first polarized proton collisions have resulted in cross section data that show fixed-order perturbative QCD is a robust framework for understanding inclusive particle production at $\sqrt{s}=200 \mathrm{GeV}$. The first spin asymmetry measurements have begun for inclusive $\pi^{0}$ production. The analyzing power at large $x_{F}$ is found to be large. Additional measurements are needed to disentangle contributions from different mechanisms. The first measurements of the double helicity asymmetry $\left(A_{L L}\right)$ for midrapidity $\pi^{0}$ production have been reported. The expectations are that significantly more precise measurements can be completed in the upcoming RHIC run. The new data will address whether gluon polarization makes significant contributions to the spin of the proton. Future measurements of $A_{L L}$ for direct photon production will become feasible as the luminosity and polarization at RHIC improves. Future runs at $\sqrt{s}=500 \mathrm{GeV}$ will measure parity violating spin asymmetries for $W^{ \pm}$ production.

\section{ACKNOWLEDGMENTS}

The progress in the RHIC spin program has been made by a very large group of people. This report is a summary of their work. Groups involved are the Collider-Accelerator De- 
partment at BNL, with primary responsibility for developing the capability to accelerate, store and collide polarized proton beams and the experiment collaborations; STAR and PHENIX, with primary responsibility for building, operating and analyzing the results from the collider detectors; and the polarimetry group that have developed techniques for measuring the beam polarization. The RHIC spin project has received important support from the the Department of Energy, the RIKEN institute and the National Science Foundation.

I would like to thank G. Bunce, A. Ogawa and G. Rakness for their careful reading of this manuscript.

\section{REFERENCES}

1. J. Blümlein and H. Böttcher, Nucl. Phys. B636,225 (2002).

2. Gerry Bunce, Naohito Saito, Jacques Soffer and Werner Vogelsang, Ann. Rev. Nucl. Part. Sci. 50, 525 (2000).

3. L. C. Bland, in Physics with a High Luminosity Polarized Electron Ion Collider, eds. L. C. Bland, T. Londergan and A. Szczepaniak (World Scientific, Singapore, 2000). Also available at hepex/9907058.

4. Ya.S Derbenev and AM. Kondratenko, Sov. Phys. JETP 37, 968 (1973); Ya.S Derbenev et al., Part. Acc. 8, 115 (1978).

5. D. P. Grosnick et al., Nucl. Instr. Meth. A290 (1990) 269.

6. J. Tojo et al., Phys. Rev. Lett. 89 (2002) 052302; O. Jinnouchi et al., AIP Conf. Proc. bf 675 (2003) 817.

7. A. Bravar et al, 16th Int. Spin Physics Symposium (SPIN 2004), to be published in the proceedings.

8. H. Okada et al, 16th Int. Spin Physics Symposium (SPIN 2004), to be published in the proceedings.

9. I. Alekseev et all, 16th Int. Spin Physics Symposium (SPIN 2004), to be published in the proceedings.

10. T. L. Trueman, hep-ph/0203013.

11. F. Aversa et al., Nucl. Phys. B327 (1989) 105; B. Jager et al., Phys. Rev. D 67 (2003) 054005; D. de Florian, ibid. 67 (2003) 054004.

12. J. Pumplin et al., J. High Energy Phys. 07 (2002) 012.

13. B. A. Kniehl et al., Nucl. Phys. B597 (2001) 337.

14. S. Kretzer, Phys. Rev. D 62 (2000) 054001.

15. S. S. Adler, et al. (PHENIX collaboration), Phys. Rev. Lett. 91 (2003) 241803.

16. J. Adams, et al. (STAR collaboration), Phys. Rev. Lett. 92 (2004) 171801.

17. C. Bourrely and J. Soffer, Eur. Phys. J. C 36 (2004) 371 and hep-ph/0311110.

18. K. Okada et al. (PHENIX collaboration), 16th Int. Spin Physics Symposium (SPIN 2004), to be published in the proceedings.

19. C. Adler et al. (STAR collaboration), Phys. Rev. Lett. 91 (2003) 172302; J. Adams et al. (STAR collaboration), Phys. Rev. Lett. 91 (2003) 072304.

20. A. Ogawa, contribution to the 12th International Workshop on Deep Inelastic Scattering (to be published), nucl-ex/0408004.

21. Daniel Boer and Werner Vogelsang, Phys.Rev. D69 (2004) 094025.

22. J. Collins, Nucl. Phys. B396 (1993) 161.

23. M. Anselmino, M. Boglione, and F. Murgia, Phys. Rev. D 60, 054027 (1999).

24. D. Sivers, Phys. Rev. D 41 (1990) 83; 43 (1991) 261.

25. M. Anselmino, M. Boglione, and F. Murgia, Phys. Lett. B 362, 164 (1995); M. Anselmino and F. Murgia, ibid. 442, 470 (1998).

26. A. Efremov and O. Teryaev, Phys. Lett. 150B (1985) 383; J. Qiu and G. Sterman, Phys. Rev. D 59 (1998) 014004; Y. Koike, AIP Conf. Proc. 675 (2003) 449.

27. F. Videbaek et al. (BRAHMS collaboration), 2004 Fall Meeting of the Division of Nuclear Physics.

28. B. E. Bonner et al., Phys. Rev. Lett. 61 (1988) 1918; D. L. Adams et al., Phys. Lett. B 261 (1991) 201; 264 (1991) 462; Z. Phys. C 56 (1992) 181; A. Bravar et al, Phys. Rev. Lett. 77 (1996) 2626. 
29. R. D. Klem et al., Phys. Rev. Lett. 36 (1976) 929; W. H. Dragoset et al., Phys. Rev. D 18 (1978) 3939; S. Saroff et al., Phys. Rev. Lett. 64 (1990) 995; B. E. Bonner et al., Phys. Rev. D 41 (1990) 13; K. Krueger et al., Phys. Lett. B 459 (1999) 412; C. E. Allgower et al., Phys. Rev. D 65 (2002) 092008. 30. S. S. Adler, et al. (PHENIX collaboration), Phys. Rev. Lett. 93 (2004) 202002.

31. D. L. Adams et al., Phys. Lett. B261 (1991) 197.

32. B. Jäger et al., Phys. Rev. D 67 (2003) 054005.

33. M. Glück et al., Phys. Rev. D 63 (2001) 094005. 